\title{
State of the art and challenges in sequence based T-cell epitope prediction
}

\author{
Claus Lundegaard ${ }^{1 *}$, Ilka Hoof ${ }^{2}$, Ole Lund ${ }^{1}$, Morten Nielsen ${ }^{1}$
}

\begin{abstract}
Sequence based T-cell epitope predictions have improved immensely in the last decade. From predictions of peptide binding to major histocompatibility complex molecules with moderate accuracy, limited allele coverage, and no good estimates of the other events in the antigen-processing pathway, the field has evolved significantly. Methods have now been developed that produce highly accurate binding predictions for many alleles and integrate both proteasomal cleavage and transport events. Moreover have so-called pan-specific methods been developed, which allow for prediction of peptide binding to MHC alleles characterized by limited or no peptide binding data. Most of the developed methods are publicly available, and have proven to be very useful as a shortcut in epitope discovery. Here, we will go through some of the history of sequence-based predictions of helper as well as cytotoxic T cell epitopes. We will focus on some of the most accurate methods and their basic background.
\end{abstract}

\section{Challenges from infectious diseases}

From the dawn of life, there has been a constant risk of infection by foreign organisms so that only host organisms that have developed an effective protection against these pathogens survived through evolution. On the other hand, this has put an evolutionary pressure on the pathogenic organisms to circumvent the developed protection mechanisms. Especially single-celled organisms and viruses, which generally have a relatively short generation time occasionally combined with a high mutation rate, have succeeded in finding loopholes in the protection. This million-year old arms race has led to the development of a defense system, the immune system, which itself consists of genetically diverse unicellular components that can evolve within the host organism when put under selective pressure. Occasionally, pathogens have evolved that efficiently could infect a specific host organism leading to high mortality. This is typically seen after a change of host [1]. Obviously, too high mortality among the host species would logically also lead to the pathogenic organism's own end. Due to geographic and biological barriers, such disasters generally hit only locally and were limited to

\footnotetext{
* Correspondence: lunde@cbs.dtu.dk

'The Technical University of Denmark - DTU, Dept. of Systems Biology, Center for Biological Sequence Analysis - CBS, Kemitorvet 208, DK-2800 Kgs. Lyngby, Denmark

Full list of author information is available at the end of the article
}

neighboring populations, while physically isolated populations avoided infection [2]. Today there are no longer any major restrictions on mobility and contact between human populations, which increases the small but present risk of a new pathogen posing a threat for the existing civilization. Several examples from the past few years have further exposed such threats; the SARS outbreak in 2003 did relatively quickly spread to several continents [3], and a high mortality has been observed in cases where certain strains of the avian flu, Influenza A H5N1 infect humans [4]. The recent Influenza A H1N1 pandemic, originating from pigs, is the latest example of how extensive these infections can be $[5,6]$. Fortunately, humans have recently been spared from the emergence of new pathogens that are at the same time both very contagious and extremely deadly. Chronic infections, which have little acute mortality but moderate to high mortality in longer terms are another growing problem. Examples of such are infections with hepatitis $\mathrm{C}$ virus (HCV), human immunodeficiency virus (HIV), and tuberculosis (TB).

\section{The immune system and vaccines}

The most effective protection against infections is through vaccination. Most vaccines today exist as an inactivated or more harmless form of the pathogenic organism. In several cases, there are problems with either the efficacy, side effects, or that the pathogen is 
constantly changing and thus escapes the vaccine's protection. The latter issue is one of the major obstacles to, for instance, a long lasting Influenza A vaccine. Vaccines take advantage of the features of the adaptive immune system. The immune system in general reacts to foreign substances and organisms when discovered in the body. The innate immune system gives a fast and unspecific response, which does not change with repeated occurrences of the same pathogen. The innate immune response might eliminate the intruder by itself but it also signals to the adaptive part of the immune system [7]. An existing effective humoral immunity is an extremely potent way of preventing an actual infection as the intruder will be eliminated immediately. For this reason vaccine development has traditionally been focusing on developing effective antibody responses, which can be obtained using totally inactivated pathogens, parts thereof, or even single proteins in case of vaccines against toxins such as tetanus or diphtheria [8]. However, to obtain strong and long lasting memory it appears that a strong $\mathrm{T}$ cell response is often needed [9]. The cellular arm of the immune system consists of two parts; cytotoxic $\mathrm{T}$ lymphocytes (CTL), and helper $\mathrm{T}$ lymphocytes (HTLs). Both CTL and HTL recognize peptides that are presented on the cell surface to the immune cells by the major histocompatibility complex (MHC) molecule, which in humans is referred to as the Human Leucocyte Antigen (HLA). While HTLs are needed for $\mathrm{B}$ cell activation and proliferation to produce antibodies against a given antigen, CTLs perform surveillance of the host cells and recognize and kill infected or malfunctioning cells that present non-self peptides (epitopes) [10]. In a vaccine context, the relevant proteins expressed by a given pathogen are the proteins that will be determining for a good immune response, i.e., the antigens. The part of the antigen that is recognized by the immune system is the epitope, and in the case of both the CTL and the HTL such epitopes consist of small, 8-20 amino acid long polypeptides.

\section{CTL epitopes}

In the MHC class I pathway, peptides from endogenous antigens bound to class I MHCs are presented to CTLs, which are carrying the CD8 receptor (CD8+ T cells). To be presented, a precursor peptide is usually first generated by the proteasome, a large cytosomal protease complex $[11,12]$. For further processing, the peptides must enter the endoplasmic reticulum (ER). This generally happens by active transport mediated by the transporter associated with antigen processing (TAP) [13]. However, some peptides can enter the ER even with an absent TAP function, as some presented peptides originate from proteins containing a signal peptide. These proteins may enter the ER through the Sec61 transporter complex [14] and should be considered especially when dealing with infected or malignant cells that might have an impaired TAP function $[15,16]$. This is highly relevant for peptides binding to MHCs belonging to the abundant A2 HLA serotype where TAP independent presentation is responsible for up to $10 \%$ of the A2 restricted epitopes [17]. During or after transport into the ER a potential epitope must bind to the MHC class I molecule $[18,19]$ generally facilitated by the helper protein tapasin $[20,21]$, before it can finally be presented on the cell surface. The most selective step in the classical MHC class I pathway is binding of a peptide to the MHC molecule. To be an epitope, i.e., to raise a CTL response, a peptide should generally bind with an affinity stronger than $500 \mathrm{nM}$ [22]. As a support for this general assumption, Moutaftsi et al. [23] found that of the 49 epitopes that are responsible for $95 \%$ of the total $\mathrm{CD} 8+\mathrm{T}$ cell response against a vaccinia challenge in mice $90 \%$ bind $\mathrm{MHC}$ with an affinity stronger than $500 \mathrm{nM}$. The work by Moutaftsi et al. also clearly underlines the usefulness of predictions in vaccine development, as only a very limited subset of peptides derived from the vaccinia proteome had to be tested to identify epitopes responsible for $95 \%$ of the CTL response. The tested subset included only the best $1 \%$ predicted of all the possible peptides.

\section{MHC class I binding predictions}

Since MHC binding of a peptide is a necessary requirement for its recognition by a $\mathrm{T}$ cell, predicting their capability to bind MHC molecules can facilitate and significantly cost-reduce the identification of $\mathrm{T}$ cell epitopes in a set of peptides. The majority of peptides binding to MHC class I molecules have a length of 811 amino acids, even though several longer epitopes have been identified [24]. The second position and the C-terminal position of the peptide are typically the most important for binding, and these positions are referred to as anchor positions $[25,26]$. For some alleles, the binding motifs further have auxiliary anchor positions. For example, peptides binding to the human HLAA*0101 allele have position 3 as an additional anchor $[25,27,28]$. Only few different amino acids are tolerated at the anchor positions of peptides binding to a given MHC allele. The discovery of such allele-specific motifs led to the development of the first algorithms for prediction of peptide binding [29-31], which essentially determined whether a peptide did or did not match the binding 'motif' of the MHC molecule.

As more data has accumulated, it has become possible to go beyond the match $/$ mismatch classification of a motif prediction. By use of statistical methods, scores can be calculated for each possible amino acid at each position in a peptide, leading to an Lx20 scoring matrix 
where $\mathrm{L}$ is the length of the peptide. For predictions, it is then assumed that the amino acids at each position along the peptide sequence contribute a certain binding energy (the score from the matrix), which can independently be added up to yield the overall binding energy of the peptide [32-34]. This type of approach is used by the EpiMatrix (commercial) [35], BIMAS (http:// www-bimas.cit.nih.gov/molbio/hla_bind/) [33], SYFPEITHI (http://www.syfpeithi.de/) [36], RANKPEP (http://bio.dfci.harvard.edu/RANKPEP/) [37], Gibbs sampler (http://www.cbs.dtu.dk/biotools/EasyGibbs/) [38], SMM (http://tools.immuneepitope.org/analyze/ html/mhc_binding.html) [39], and ARB (http://tools. immuneepitope.org/analyze/html/mhc_binding.html) methods [40]. These methods differ in the way they derive the matrix coefficients. Some are trained by statistical methods that analyze how often a given amino acid is seen at a given position in binding versus nonbinding peptides. Matrix coefficients can also be determined by a machine learning procedure, which aims at finding the coefficients that best explain the observed binding data. This can be done by interpreting the matrix scores for a peptide as predicted binding affinities and by minimizing the distance between predicted and measured values. This is the approach utilized by SMM, which is presently the best performing matrix method in published benchmark studies $[41,42]$.

Matrix-based methods cannot take correlated effects into account, i.e., where the contribution to the binding affinity of an amino acid at one position depends on amino acids at other positions in the peptide. Higher order methods like artificial neural networks (ANNs) and Support Vector Machines (SVMs) are ideally suited to take such correlations into account [43-48]. These methods can be trained with data either in the format of binder/non-binder classification, e.g. binders from the SYFPEITHI database of eluted peptides [36], or as real affinity data as can be found in the Immune Epitope Database (IEDB) $[49,50]$. Likewise the predictors can either be trained to output a score that correlates with the chance that a given peptide is a binder or to output a score that corresponds to a predicted affinity [51]. The ANN based predictor NetMHC $[45,47,52]$ was trained using both sequence input from affinity data mainly found in the IEDB as well as output from matrices generated by SYFPEITHI [36] eluted peptides using the Gibbs sampler approach [47]. In two recent benchmark comparisons the NetMHC-3.0 implementation was the most successful method including higher order sequence correlations $[41,42]$. The NetMHC method has been further improved in the NetMHC-3.2 version (http:// www.cbs.dtu.dk/services/NetMHC) by training on data with larger peptide and allelic coverage. (Lundegaard et al., J. Imm. Meth., submitted). As mentioned earlier, most epitopes and MHC binding peptides discovered to date are of length 8,9 , or 10 amino acid residues, even though longer epitopes have been identified, mostly hendecamers, but also a few even longer [24]. Data driven prediction-algorithms for MHC class I binding are for the most part limited to predict the same lengths as they have been trained on, and in the IEDB, very few examples of such longer peptides exist today. Of all unique eluted MHC binding peptides in the current version of the IEDB database, only $10 \%$ are longer than 10 residues and $4 \%$ are longer than 11 . Some MHC:peptide binding methods have been developed using the information of the three dimensional structure of known complexes. These methods should in principle be able to predict binding also of longer peptides. However, not even on nonamer peptides are these methods as accurate as the data driven methods [53,54], and have to our knowledge not been benchmarked on longer peptides. It has been shown, though, that predictions from methods trained on nonamer peptides can be used to predict the affinity of longer peptides, which has been benchmarked with peptides of a length up to 11 residues [55]. This system has been implemented into the NetMHC method. To summarize: of the prediction methods publicly available online, the neural network based NetMHC performs best on the tested evaluation sets, followed by the matrix based $S M M[41,42]$. The $S M M$ training and prediction code is freely available [39]. The implementation of online consensus MHC class I prediction tools is currently in progress at the IEDB site (Björn Peters, personal communication), as an approach of combining different prediction methods might give even better results [56]. How accurate the best of methods are can be exemplified by comparing the prediction accuracy of the single methods with the correlation between different experimental methods [42]. Both the $S M M$ and the NetMHC methods are available via the IEDB website (http://www.immuneepitope.org) [56], and NetMHC is additionally accessible from http://www.cbs.dtu.dk/services/NetMHC.

Today more than 2000 HLA alleles have been identified, and as they in principle bind different peptide repertoires, the task of mapping the peptide preferences for each and every one of these would be experimentally overwhelming. Initially only the most common alleles were examined, but it was soon clear that some alleles were sharing peptide preferences often, which did not always correlate with the amino acid sequence similarity of the compared alleles [57]. This discovery lead to the concept of supertypes, where several alleles are clustered into groups (supertypes), based on the degree of functional similarity (Figure 1) [57-63]. In this approach still only the most common alleles were studied, however, the population coverage of identified epitopes could be 


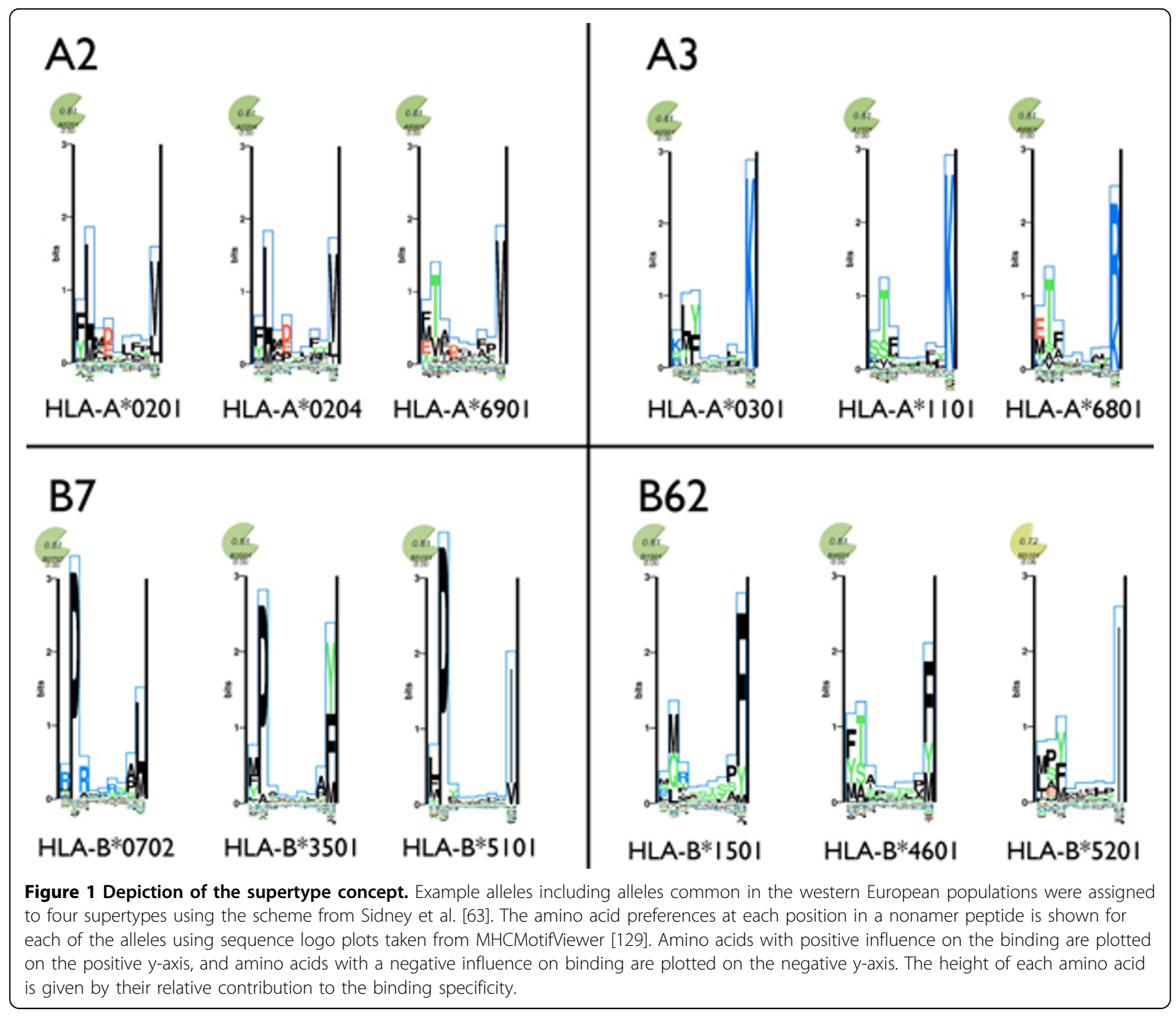

theoretically extrapolated assuming complete peptide binding overlap between alleles within a given supertype.

Lately, the amount of publicly available binding data has increased significantly mainly due to the huge effort funded by NIH resulting in the IEDB database [49]. This database is now very extensive both in terms of the number of different peptides and the number of different MHC alleles for which binding data exist. Furthermore, the MHC class I binding data are very homogeneous in quality as more than $99 \%$ of the quantitative binding data in the IEDB database generated since 2006 were generated by two comparable assays developed in the laboratories of A. Sette and S. Buus [64-67]. More than 95\% of the class I data has been generated since 2005, and less than $2 \%$ before 2001 . However, besides leading to MHC prediction systems, which now cover a large number of different HLA alleles
$[42,68]$, this large growth in the amount of MHC peptide-binding data has enabled the development of new so-called pan-specific algorithms. These pan-specific methods go beyond the conventional single allele approach and are able to predict peptide-binding to HLA alleles, for which the sequence is known but only limited or no experimental binding data are available [68-73]. The architecture of the training system of NetMHCpan has been outlined in a way that takes both the peptide sequence and the MHC contact environment into account (Figure 2). Polymorphic positions in the MHC assumed to be in contact with a residue in a bound peptide have been mapped in order to extract a pseudo sequence representing the given $\mathrm{MHC}$ molecule [72]. This pseudo sequence is used as input in the training coupled with a peptide sequence and the measured affinity of the given peptide:MHC. Thus, the machine 

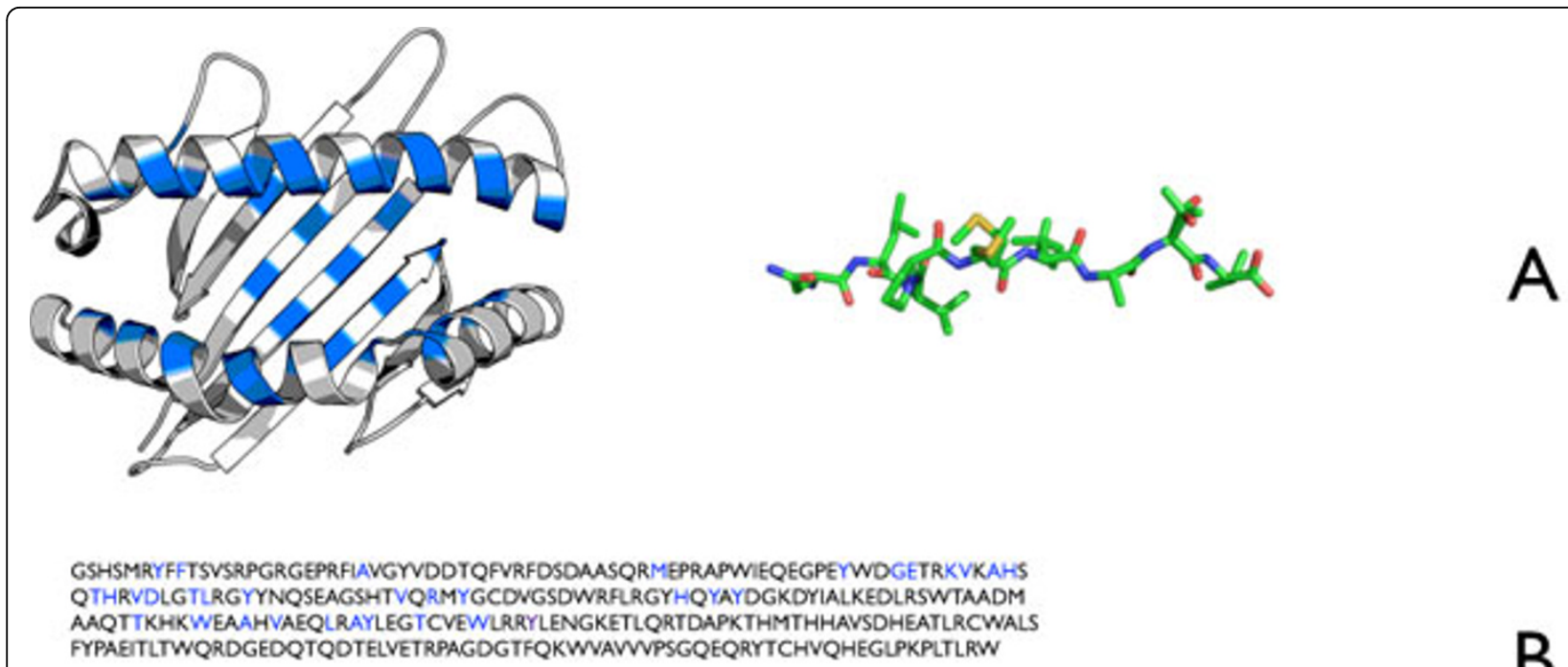

\begin{tabular}{|c|c|}
\hline HLA & Aff \\
\hline$A \theta 2 \theta 1$ & 0.131751 \\
\hline$A \theta 2 \theta 1$ & $\theta, 4875 \theta \theta$ \\
\hline$A \theta 2 \theta 1$ & 0.364186 \\
\hline$A 0201$ & 0.582749 \\
\hline$A \theta 2 \theta 1$ & $\theta .2067 \theta 0$ \\
\hline$A \theta 2 \theta 1$ & $\theta .727865$ \\
\hline$A \theta 2 \theta 2$ & $\theta .706274$ \\
\hline$A 0203$ & 1.000000 \\
\hline$A 0206$ & 0.682619 \\
\hline A6802 & $\theta .407855$ \\
\hline
\end{tabular}

PePtIde
WLQQHSIA
SQVSFQQPL
SQCQAIHNV
LQQSTYQLV
LQPFLQPQL
VLAGLLGNV
VLAGLLGNV
VLAGLLGNV
VLAGLLGNV
VLAGLLGNV

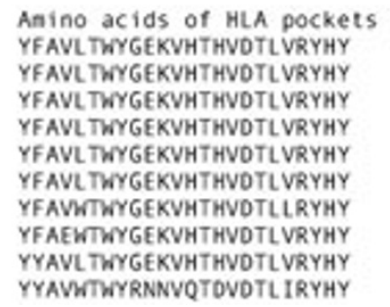

$$
\begin{array}{ll}
A \theta 2 \theta 1 & \theta .131751 \\
A \theta 2 \theta 1 & \theta .4875 \theta \theta \\
A \theta 2 \theta 1 & \theta .364186 \\
A \theta 2 \theta 1 & 0.582749 \\
A \theta 2 \theta 1 & 0.2067 \theta \theta \\
A \theta 2 \theta 1 & \theta .727865 \\
A \theta 2 \theta 2 & \theta .706274 \\
A \theta 2 \theta 3 & 1.00000 \theta \\
A 02 \theta 6 & 0.682619 \\
A 68 \theta 2 & \theta .407855
\end{array}
$$

Figure 2 Description of the NetMHCpan approach. A) Amino acids used for prediction are residues from the MHC alpha chain that are found to be in contact with the peptide using structural data (blue in left) and the full binding peptide (right). B) The identified MHC residues in the amino acid sequence of HLA-A ${ }^{*} 0201$ are labeled blue. C) The labeled residues from B presented as a pseudo sequence (left) and the peptide sequence (right). D) Pairs of peptide sequences (left) and pseudo sequences (second from left) are presented to the ANN with the experimentally determined log scaled affinity (far right). The displayed allele information is not an input to the ANN. During the training the weights are adjusted in order to minimize the error between predicted output and the assigned affinity.

learning method behind the predictions is trained to be able to combine the information provided by the MHC sequence and the peptide sequence in order to predict a specific binding affinity. In this way, the system can combine information from the MHC sequence with the peptide sequence to derive cross correlations and is able to predict the outcome of MHC:peptide combinations that it has not encountered during the training. Several pan-HLA methods have been evaluated in a large-scale benchmark, and the outcome of this evaluation demonstrated the power of the pan-specific methods. Not only do these methods predict peptide-binding affinities to previously uncharacterized MHC molecules but the incorporated training setup also boosts the predictive performance for already characterized alleles by leveraging information from neighboring $\mathrm{MHC}$ molecules [74], see Table 1. Kiss [70] is available from http://cbio.

Table 1 Performance of available pan-specific predictors

\begin{tabular}{lllll}
\hline Performance Measure & Kiss & ADT & NetMHC & NetMHCpan \\
\hline Pearson CC & 0.455 & 0.488 & 0.593 & 0.620 \\
Spearmans Rank CC & 0.44 & 0.522 & 0.561 & 0.600 \\
AUC & 0.734 & 0.756 & 0.807 & 0.820 \\
\hline
\end{tabular}

Performance values taken from [74]. The prediction servers have been evaluated on a set of binders to 17 HLA-A alleles and 16 HLA-B alleles. The data had not been used for training of any of the tested alleles. 
ensmp.fr/kiss/, $A D T$ [71] is available at http://atom. research.microsoft.com/hlabinding/, and NetMHCpan $[72,73]$ is available at http://www.cbs.dtu.dk/services/ NetMHCpan. The latter server has implemented the approach of extrapolating from 9mers to prediction of binding for peptides up to 11 residues in length [55] and allows prediction for all known HLA-A, -B, and -C alleles, as well as some non-human primate, mouse and pig MHC alleles.

In an attempt to perform a completely unbiased benchmark of different MHC binding prediction approaches, several groups have participated in a competition that has been held in connection with the ICANN 09 conference (http://www.kios.org.cy/ ICANN09/MLI.html). The binding to the MHC alleles HLA-A*0101, HLA-A*0201, and HLA-B*0702 were to be predicted for a total of 17710 mer peptides and 265 9 mers. The results of this competition placed NetMHC3.2 and NetMHCpan-2.2 as the best performing methods on the benchmark set, and a prediction approach using the simple mean of the predictions from these two methods was awarded the first price among the 20 competing methods (Vladimir Brusic, personal communication, submitted to J. Imm. Meth.).

\section{Prediction of other MHC class I pathway events}

In the following, we describe predictions of proteasomal cleavage and TAP binding. The proteins responsible for these events are basically monomorphic, and developers of prediction methods do not face the same allele problem as is present for MHC binding prediction. This should in principle make the task of developing accurate prediction methods easier. This is, however, not the case as the assays determining the cleavage and binding are not developed for high throughput to the same extent as is the case for MHC:peptide binding assays. For this reason data for these two processing events are in general scarce.

The complex enzymatic specificity of the proteasome makes the prediction of its cleavage patterns highly challenging. The proteasome comprises multiple catalytically active sites, each with a distinct specificity [75,76]. A further complication is that two versions of the proteasome exist. The proteasome that functions in most cells and which has the main task of recycling superfluous or malfunctioning proteins is constitutively expressed and is therefore called the constitutive proteasome. An inducible version of the proteasome, the immunoproteasome, is expressed when a cell receives signals from the innate or the adaptive immune system indicating that it should enter an 'alarm' state. The immunoproteasome has catalytic subunits with different specificity than the constitutive proteasome. This change gives rise to a catalytic complex, which cleaves proteins into fragments that are better processed by the other players in the MHC class I pathway [77]. The outcome of proteasomal cleavage has been considered in two separate ways when it comes to predictive purposes. One way is to predict the chance of a given position in the protein sequence to be cleaved. Another approach is to predict the likelihood that a given peptide fragment will arise after proteasomal cleavage. FragPredict, which is publicly available as a part of MAPPP service (http:// www.mpiib-berlin.mpg.de/MAPPP/), takes the latter approach and consists of two sequential algorithms. The first algorithm uses a statistical analysis of cleavageenhancing and -inhibiting amino acid motifs to predict potential proteasomal cleavage sites [78]. The second algorithm predicts the likelihood that a given peptide fragment will arise using the results of the first algorithm as an input. The second algorithm has been developed to select the most likely fragments to be generated. The model calculates the time-dependent degradation based on a kinetic model of the 20S proteasome [79]. The PAProC (http://www.paproc.de) method predicts in vitro proteasomal cleavages performed by human and wild type and mutant yeast proteasomes. The influence of different amino acids at different positions is determined by using a stochastic hill-climbing algorithm [80] based on the experimentally verified in vitro cleavage and non-cleavage sites [81]. A weight matrix method has been developed which predicts both constitutiveand immunoproteasomal cleavage specificity [82] trained on the very limited in vitro proteasomal digest data available. The NetChop [83] method has been trained using information from $\mathrm{C}$ termini of naturally processed MHC class I ligands. No other significant endopeptidases or exopeptidases processing the $\mathrm{C}$-terminus of peptides have been observed in the cell compartments involved in the class I pathway. Therefore the C-termini of MHC I presented peptides are believed to be created by proteasomal cleavage. Since some of these ligands are generated by the immunoproteasome and some by the constitutive proteasome, such a method should predict the combined specificity of both forms of proteasomes. NetChop-2.0 was evaluated to be the best-performing predictor on an independent evaluation set [84]. The SVM based Pcleavage proteasomal cleavage predictor, which is available online, has a published performance comparable to that of NetChop-2.0 [85]. An update of the NetChop method to version 3.0 [77] consists of a combination of several ANNs, each trained using a different sequence-encoding scheme of the data. NetChop3.0 (http://www.cbs.dtu.dk/services/NetChop) has an increased sensitivity as compared to NetChop-2.0, without lowering the specificity. A method using SVM predictions and apparently achieving very good results has recently been published [86]. In their evaluation, 
however, the developers do not compare AROC/AUC values, described by [87], which is the best suitable value for comparison of the performance of these kind of predictors [53]. The method is not available as software, code, or server, and still awaits independent evaluation. Finally, a new method predicting the likelihood that a given peptide originates from proteasomal cleavage has been implemented as a publicly available server (http://peptibase.cs.biu.ac.il/PepCleave_II/) [88], and according to the published evaluation this method works well. Good benchmarks for a comparison of the usefulness of the different types of predictions have not yet been implemented.

Relatively few methods have been developed to predict the specificity of TAP. Daniel et al. [89] have developed ANNs using 9-mer peptides, for which the TAP affinity was determined experimentally. Surprisingly, they found that some MHC alleles such as alleles belonging to the HLA-A*02 family have some natural ligands with very low TAP affinities. This could either be because TAP ligands can be trimmed in the ER before binding to MHC molecules [90] and that a TAP ligand therefore often enters the ER as a precursor to the MHC binding peptide, or it could be due to alternative entrance routes, as described earlier. Peters et al. [91] used an SMM based matrix to predict TAP affinity for peptides of length 9 or longer.They used this model to show that natural A2 ligands are well transported by TAP in form of precursor peptides, hence confirming the trimming hypothesis by Fruci et al. A number of different TAP binding prediction methods have since been published as recently reviewed $[54,92]$. Several methods utilizing machine-learning algorithms have been published with a predictive performance superior to the SMM method. It must be mentioned, though, that these methods probably suffered from overtraining, and only a single SVM based method, TAPREG (http://imed.med. ucm.es), appears to have been able to match the predictive performance of the SMM based method using a new benchmark dataset [93]. However, while TAPREG works only for nonamer peptide predictions, the SMM based method was further generalized to work on peptides that are longer than 9 amino acids. It was found that mainly the three $\mathrm{N}$-terminal residues and the $\mathrm{C}$ terminal residue had influence on the binding affinity of TAP binding peptides [91,93]. Thus, the affinity of peptides longer than 9 amino acid residues can be predicted by using matrix scores only for the three $\mathrm{N}$ terminal residues and the $C$ terminal residue in the peptide.

The action of ERAAP peptidase has also been shown to be important for peptide binding [24,94], and the importance of tapasin in the class I presentation pathway has recently become evident $[20,21,95,96]$. Data regarding these players are still very scarce and their function has not been examined in relation to epitope prediction, but it is likely that methods for prediction of these events will be developed as data become available.

\section{Integrated CTL epitope predictions and optimal population coverage}

Although predictions of MHC binding in itself can be used to rank the possible CTL epitopes quite accurately $[97,98]$, even better predictions should be attainable if other steps in the antigen processing and presentation pathway were modeled and included in a final prediction. Several attempts have been made to predict the outcome of two or more steps involved in antigen processing and presentation: MAPP (http://www.mpiibberlin.mpg.de/MAPPP/) [99], NetCTL (http://www.cbs. dtu.dk/services/NetCTL) [100], NetCTLpan (http://www. cbs.dtu.dk/services/NetCTLpan), MHCpathway (http:// tools-int-01.liai.org/analyze/html/mhc_processing.html) [101], EpiJen (http://www.darrenflower.info/EpiJen/) [102], and WAPP (http://www-bs.informatik.uni-tuebingen.de/Services/WAPP) [103]. All of these methods attempt to predict antigen presentation by integrating peptide:MHC binding predictions with one or more of the other events involved in the antigen presentation pathway. How well do these methods perform, and which of the methods work best? In a benchmark, a set of verified epitopes can be used as the positive data set. But having only positive data, it is only possible to get a sensitivity score, and methods that will predict any peptide as an epitope will reach the highest rank. On the other hand, a negative data set (containing peptides that cannot induce an immune response) is difficult to define because it is impossible to guarantee that a peptide will never be an epitope in any individual expressing a given HLA allele. To circumvent this problem, epitopes from extensively studied pathogens, such as HIV, are often used as the positive set, and all other peptides that are present in the whole proteome of the same pathogen and have never been shown to give an immune response are chosen as the negative set (non-epitopes), thus assuming that they will at least have a very low probability of being epitopes. A comparison has been published calculating the predictive performance of several publicly available MHC-I presentation prediction methods [104]. The outcome, using such a large-scale benchmark on known HIV epitopes (http://www.cbs.dtu.dk/ suppl/immunology/CTL-1.2/HIV_dataset) revealed that the NetCTL and MHCpathway methods were ranked the most accurate with $>75 \%$ of the epitopes ranking among the top $5 \%$ peptides sorted by the prediction score [104]. The majority of the described methods only work for a limited number of MHC alleles. To date only the NetCTLpan method has integrated the described pan-specific MHC binding prediction systems with 
predictions of proteasomal cleavage and TAP translocation [98].

When testing predicted epitopes for response in patients or donors the success rate is around 10\% depending on selected cutoff and pathogen $[23,105,106]$. Since the affinity predictions are far more accurate than this there might be other issues to address. These could be inherent issues such as stability of the peptide:MHC complex (half life), the influence of tapasin on successful MHC loading, MHC competition, or holes in the T cell repertoire. But also the fact that many pathogens interfere with the players in the classical MHC class I pathway might influence the epitope repertoire [15]. The outline and outcome of a selected set of epitope discovery experiments have recently been reviewed elsewhere [51].

\section{Helper T cell epitopes}

Helper $\mathrm{T}$ cells with a $\mathrm{T}$ cell receptor (TCR) specific for antigen-derived peptides must be activated to get strong $B$ cell responses [107]. The epitope recognized by a helper TCR is usually somehow connected to the epitope that is recognized by the $\mathrm{B}$ cell receptor, but the two different receptors do not necessarily recognize overlapping epitopes or even epitopes from the same protein. $\mathrm{T}$ cells can recognize internal peptides that do not need to be a part of the surface-surface interactions with the B cell receptor. HTLs, which normally carry the $\mathrm{CD} 4$ receptor and are therefore also called CD4+ T cells, recognize peptides presented by the MHC class II molecule on the surface of professional antigen presenting cells such as macrophages, dendritic cells, and B lymphocytes. Peptides presented by class II MHCs usually originate from internalized proteins, thus, class II peptide presentation follows a different path than the MHC class I presentation pathway [108]. In short, MHC class II molecules associate with the invariant chain (Ii) in the ER and the MHC:Ii complexes accumulate in endosomal compartments. Here, Ii is degraded, while another MHC-like molecule which in humans is called HLA-DM, loads the MHC class II molecules with the best available ligands originating from endocytosed antigens that have been degraded in the lysosomes partly simultaneously with the MHC maturation process. The peptide:MHC class II complexes are subsequently transported to the cell surface for presentation.

In contrast to MHC class I, peptide affinity data for MHC class II have been generated using a diverse set of experimental assays by a large number of different groups. About $80 \%$ of the quantitative data has been produced using one single assay type, whereas 20 groups using more than five different assay types produced the remaining $20 \%$. Less than $80 \%$ of the data were produced after 2006 , and more than $15 \%$ of the data were produced before 2001 [109]. Most binding data describing the specificity of MHC molecules are equilibrium binding affinity values. Binding affinity might not be the only relevant feature for the characterization of epitopes. Binding stability might be equally relevant because the avidity of the MHC peptide complex to bind $\mathrm{T}$ cells clearly depends both on the equilibrium binding constant and the stability of the complex. Complementing the MHC binding data with peptide stability measurements may, thus, lead to improved epitope predictions. As a result of the open ends of the MHC class II binding cleft, peptides may bind in multiple registers. Several conflicting studies have shown both positive and negative effects of including such multiple binding registers into the prediction of MHC class II binding, and no consensus has been reached in the field as to how big the effect of multiple binding registers would be for an accurate description of the binding specificity. Finally, for naturally processed MHC ligands and CD4 epitopes, factors other than peptide-MHC binding can influence the peptide immunogenicity, including susceptibility to proteolytic activity in the endosome/lysosome and peptide/antigen abundance in the antigen-presenting cell.

\section{MHC class II binding predictions}

Unlike MHC class I molecules, the binding cleft of MHC class II molecules is open-ended [110], which allows for the bound peptide to have significant protrusions at both ends. As a result MHC class II binding peptides have a broader length distribution typically of eleven to twenty residues [111]. However, the majority of the binding interaction is mediated by a 9 amino acid residue core sequence of the bound peptide. This complicates binding predictions, as the identification of the correct alignment of the binding core is a crucial part of identifying the MHC class II binding motif $[38,56]$. Identifying this core is difficult, as the MHC class II binding motifs have relatively weak and often degenerate sequence signals. The majority of MHC class II binding prediction methods are based on the assumption that the peptide-MHC binding affinity is determined solely from a nine amino acid binding core motif. An early effort, TEPITOPE developed by Jürgen Hammer [112], used this assumption. The data were obtained by phage display and binding to a selected set of HLA-DRB1 molecules with a changing central 9mer core of the presented peptide. Position specific scoring matrices (PSSM) were derived using statistical analysis of the amino acids observed at each position in binding versus non-binding peptide cores. Such PSSMs were generated for a number of selected HLA-DRB1 alleles and, using structurally derived data, the anchor positions in the peptides were associated with certain binding pockets in the MHC molecule. Assuming that these binding pockets were mutually independent, virtual PSSMs for 
HLA-DRB1 alleles, for which no data was available, were created by matching amino acid pocket residues of the uncharacterized allele to pockets for the alleles with characterized binding motif. For a long time, this method was the best method for MHC class II binding prediction. Since TEPITOPE was originally only made available for PC use, the PSSMs were later derived from publications and made publicly available as a part of the web accessible class II predictor ProPred (http://www. imtech.res.in/raghava/propred/) [113]. Even though binding data became available for naturally processed peptides, e.g., from SYFPEITHI [36] it proved difficult to make prediction systems that significantly exceed the accuracy of TEPITOPE/ProPred. One of the major obstacles has been the identification of the 9mer binding core within these generally longer peptides. Several attempts have been made using more sophisticated methods such as Gibbs sampling [38] or SVMs [114]. The assumption that binding can be predicted from a 9 mer core alone is clearly an oversimplification as it is known that peptide flanking residues (PFR) on both sides of the binding core may contribute to the binding affinity and stability of the peptide:MHC complex [115]. Some methods for MHC class II binding have attempted to include PFRs indirectly, in terms of the peptide length, in the affinity prediction [116]. Later, it was demonstrated that including PFRs in MHC class II predictions does in fact improve the prediction accuracy [117]. The method SMM-align (http://www.cbs.dtu.dk/ services/NetMHCII-1.1), which implements this approach, has been shown to perform best by independently conducted validations [56,118]. Most of the methods for MHC class II binding predictions have been trained and evaluated on very limited data sets covering only a single or a few different MHC class II alleles, making it very difficult to compare the different performance values and establish generality of the methods. A recent large-scale comparison of prediction methods for MHC class II binding [56] covered 14 HLA-DR (human MHC) and two mouse class II alleles. Recently, an ANN-based method, NN-align (http:// www.cbs.dtu.dk/services/NetMHCII-2.2), has been published [119] as an extension to the SMM-align method. As depicted in Figure 3, the NN-align method uses the current weights optimized in the previous training round to select the optimal 9mer core and PFRs for each of the peptides within the training set. The ANN weights are then optimized on the basis of the errors between the predicted binding affinity using the newly defined core and PRFs. Now, the cores and PFRs are in turn redefined based on the new weights and the iteration is continued until the error ceases to decrease on an external part of the training set not used to optimize the weights. This method works significantly better than previously published methods, but awaits external independent evaluation. Besides the previously described 14 HLA-DR alleles, the updated NetMHCII-2.2 method includes prediction for six of the most common HLADQ and DP alleles.

In a way the TEPITOPE approach was already an early pan-specific predictor, but class II predictions have now further benefited from the increasing number of data points available, both regarding the number of peptides and alleles. Pan-specific predictors have been developed covering all HLA-DRB alleles [120] and as the amount of data increases this trend will likely proceed to the other class II loci, DQ and DP.

Even though significant improvements have been made on MHC class II:peptide binding predictions, we are still far from the accuracy obtained in class I predictions. Regarding the usefulness of class II predictions, the lower accuracy is to some extent compensated for by the fact that longer peptides can be used (containing a higher number of possible epitopes each) and that class II MHCs are more promiscuous.

A number of epitope discovery experiments have been performed were MHC class II binding predictions, mainly TEPITOPE/ProPred, have been included as a filtering step [109,121]. As another example of a recent Th1 epitope discovery effort where MHC class II binding predictions have been integrated is the work of S.A. Mustafa [122]. Here, it was shown that MPT63, a major secreted protein of Mycobacterium tuberculosis, induced moderate Th1 cell reactivity. Analysis of MPT63 hosted peptides for binding to 51 HLA-DR alleles, using ProPred, showed that MPT63 sequences could bind to all the 51 alleles, and nine of the ten peptides of MPT63 were predicted to bind promiscuously.

\section{Selection of an optimal epitope pool}

Searching for potential $\mathrm{T}$ cell epitopes can be guided using in silico screening procedures as explained above. Genome wide screening procedures will often identify thousands of potential epitope candidates caused by genomic diversity of the pathogen and the HLA allelic diversity of a given host population. Due to economic and practical limitations, only a small set of epitope candidates can be handled in subsequent epitope validation assays. Several methods have been published recently aiming at identifying a peptide subset that will provide optimal pathogen genomic and HLA coverage in a given population [105,123-125]. The method by Fischer et al. aims at designing mosaic protein with maximal 9-mer peptide coverage of the pathogen genomic diversity. The EpiSelect method described by Perez et al. [105] aims at identifying sets of CTL epitopes with maximum coverage of the genomic variation of the pathogen. All available variants of an organism of interest are screened for 


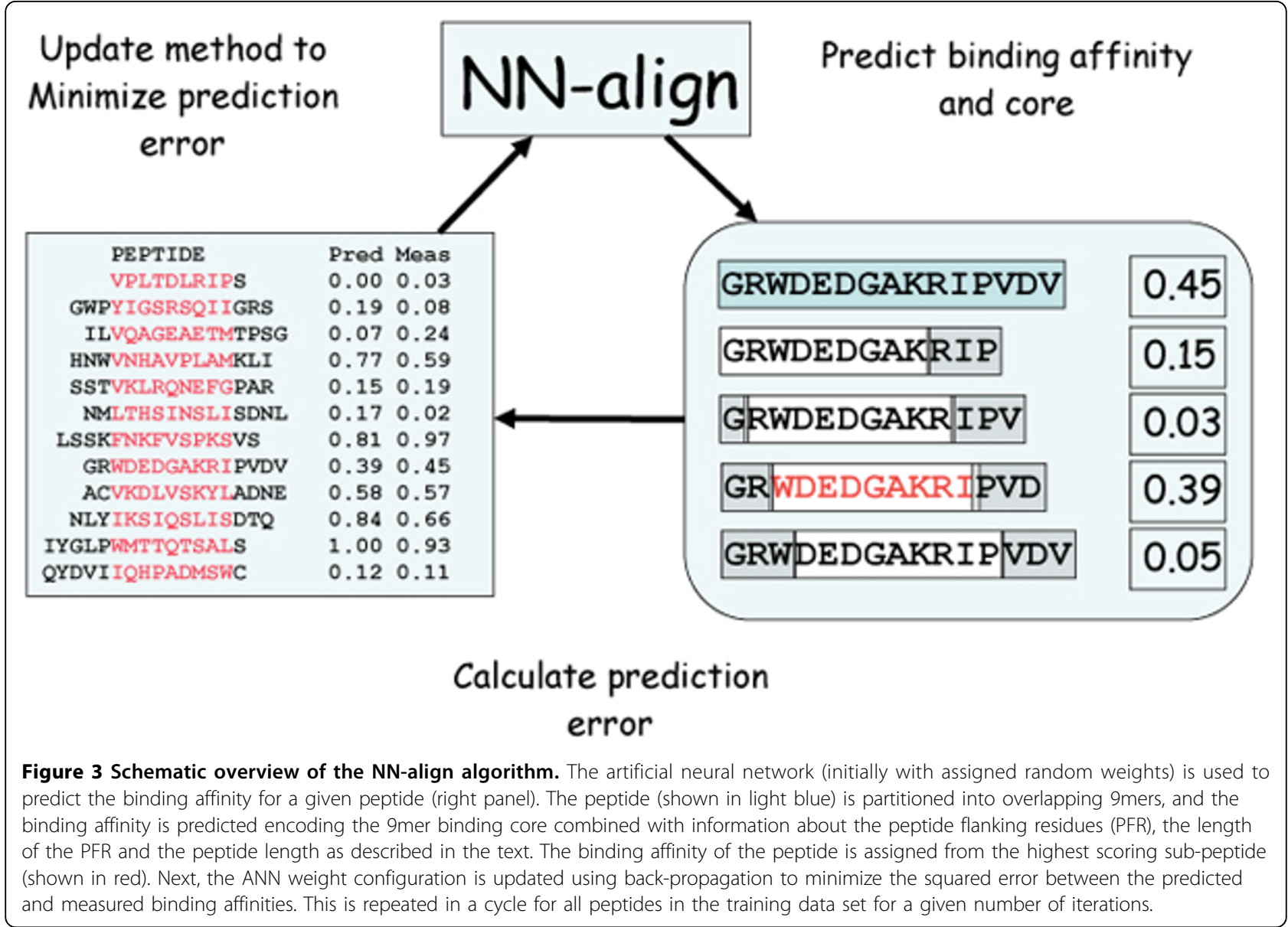

peptides predicted to bind to a given allele or supertype representative. The peptide-binder predicted to be present in most of the variable pathogenic strains is selected first. In repetitive selection rounds, new predicted binders are selected according to a scheme that maximizes the overall coverage of the pathogenic strains and leaves as few strains as possible uncovered. This algorithm thus goes one step further than the method by Fischer et al. [123], and includes the HLA restriction in the peptide selection. In the published study, epitopes were predicted for allele representatives of 9 supertypes using NetCTL. For each of the supertypes, peptides were consecutively selected by the EpiSelect scheme. Of 184 peptides tested against blood monocytes from 31 HIV patients infected with various HIV subtypes 114 (62\%) were recognized by at least one study subject, and 45 were novel epitopes. Using the EpiSelect algorithm, Perez et al. [105] were able to demonstrate how it is possible to detect and evaluate both the magnitude and breadth of epitope-specific CTL responses in a genetically diverse population infected with different HIV subtypes using a very limited set of HLA class I supertyperestricted epitopes, thus demonstrating the high power of these methods. An alternative approach was taken in the work by Toussaint et al. [125] where a set of peptides with maximum likelihood of eliciting a broad and potent immune response was selected from a userdefined set of predicted or experimentally determined epitopes covering different HLA alleles and pathogen genomic variants.

\section{Conclusions}

Almost two decades ago, MHC peptide-binding data were available for only a few human and mouse alleles. Even from this scarce amount of data, it was found that prediction of new potential epitopes could be performed with a decent accuracy. The large polymorphism of the MHC genomic region and especially of the MHC genes themselves became more and more clear. This challenged the usefulness of identified epitopes as vaccines since many epitopes would be needed to cover a reasonable part of a given population, which would require tremendous resources to be invested in the experimental validation of the predicted epitopes. For more than a decade, the supertype concept has been a highly valuable tool for limiting the number of epitopes needed in 
an epitope-based vaccine with broad population coverage. However, recent studies have demonstrated that supertypes do provide a strong oversimplification of the peptide binding diversity of the different MHC molecules, and that different MHC alleles within a given supertype often will restrict very different peptide repertoires $[126,127]$. To entail a detailed understanding of which $\mathrm{T}$ cell epitopes can be restricted by a given host, it has therefore become apparent that full HLA typing is required in combination with the recent advances in pan-specific MHC class I binding predictions. Several large scale studies have demonstrated that based on such detailed information, the vast majority of positive $\mathrm{T}$ cell responses can be explained $[128,129]$. These studies also underline that supertype associations may lead to poor or even wrong interpretations of the observed immune correlates.

Despite the great advances in the accuracy and allelic coverage of methods for prediction of peptide binding to MHC molecules, a great proportion of recent papers published on the subject of rational $\mathrm{T}$ cell epitope discovery apply relatively ancient methods like BIMAS and SYPHITHI for MHC class I and TEPITOPE/ProPred for MHC class II [109]. This is surprising because many benchmark studies have shown that state-of-the-art data-driven methods significantly outperform these older methods also when it comes to identification of MHC ligands and $\mathrm{T}$ cell epitopes.

It is apparent that at present MHC:peptide binding in silico models can significantly enhance the outcome of epitope discovery experiments. However, there is no doubt that human interpretation by experienced immunologists is necessary in order to correctly interpret and validate the outcome of such prediction systems. Today the most fruitful work seems to be done in collaborations between experimentalists and bioinformaticians.

The CTL epitope prediction algorithms are today at a level of accuracy where they have already been proven useful in high throughput and full genome based epitope discovery. This gives hope that the methods themselves can be used as analytic tools for investigations of systems biology nature e.g., host/pathogen interactions, and simulate the development of the immune system under specific stimuli. We do strongly believe that in the near future the number of MHC class II binding data will increase significantly, which will lead to the development of new predictive methods and will enhance the performance of existing methods. Furthermore, ongoing experiments indicate, that class II predictions, even at the current level, can be of significant help in Th epitope discovery efforts (Annika Karlsson, personal communication).

\section{List of abbreviations}

HCV: hepatitis C virus; HIV: human immunodeficiency virus; TB: tuberculosis; CTL: cytotoxic T lymphocytes; HTL: helper T lymphocytes; MHC: major histocompatibility complex; HLA: Human Leucocyte Antigen; ER: endoplasmic reticulum; TAP: transporter associated with antigen processing; ANN: artificial neural networks; SVM: support vector machine; IEDB: immune epitope database; TCR: T cell receptor; PSSM: position specific scoring matrix; PFR: peptide flanking region.

\section{Acknowledgements}

The publication of this article has been funded by NIH contract \# HHSN272200900045C

This article has been published as part of Immunome Research Volume 6 Supplement 2, 2010: Computational Vaccinology: State-of-the-art Assessments. The full contents of the supplement are available online at http://www.immunome-research.com/supplements/6/S2.

\section{Author details}

${ }^{1}$ The Technical University of Denmark - DTU, Dept. of Systems Biology, Center for Biological Sequence Analysis - CBS, Kemitorvet 208, DK-2800 Kgs. Lyngby, Denmark. ${ }^{2}$ Utrecht University, Theoretical Biology/Bioinformatics, Padualaan 8, 3584 CH Utrecht, The Netherlands.

\section{Authors' contributions}

$\mathrm{CL}$ wrote the initial draft and $\mathrm{MN}, \mathrm{OL}$, and $\mathrm{IH}$ contributed to the development and improvement of the manuscript by rewriting paragraphs and writing significant additions to the text. All authors read and approved the final manuscript.

\section{Competing interests}

Individuals of the author group have within the last five years been funded by the European Commission (LSHBCT-2003-503231, LSHB-CT-2004-012175) and the US National Institutes of Health (HHSNN26600400006C, HHSN266200400025C, HHSN266200400083C, HHSN272200900045C). No other financial or non-financial competing interests to be declared.

Published: 3 November 2010

\section{References}

1. Heeney $\mathrm{J}$ : Zoonotic viral diseases and the frontier of early diagnosis, control and prevention. J Intern Med 2006, 260:399-408.

2. White PJ, Norman RA, Trout RC, Gould EA, Hudson PJ: The emergence of rabbit haemorrhagic disease virus: will a non-pathogenic strain protect the UK? Philos Trans R Soc Lond B Biol Sci 2001, 356:1087-1095.

3. Perlman $\mathrm{S}$, Netland J: Coronaviruses post-SARS: update on replication and pathogenesis. Nat Rev Microbiol 2009, 7:439-450.

4. de Wit E, Kawaoka Y, de Jong MD, Fouchier RAM: Pathogenicity of highly pathogenic avian influenza virus in mammals. Vaccine 2008, 26(Suppl 4): D54-D58.

5. Lister P, Reynolds F, Parslow R, Chan A, Cooper M, Plunkett A, Riphagen S, Peters M: Swine-origin influenza virus H1N1, seasonal influenza virus, and critical illness in children. Lancet 2009, 374:605-607.

6. Boni MF, Manh BH, Thai PQ, Farrar J, Hien TT, Hien NT, Kinh NV, Horby P: Modelling the progression of pandemic influenza $A(\mathrm{H} 1 \mathrm{~N} 1)$ in Vietnam and the opportunities for reassortment with other influenza viruses. BMC Med 2009, 7:43.

7. Akira S, Uematsu S, Takeuchi O: Pathogen recognition and innate immunity. Cell 2006, 124:783-801.

8. Ellis RW: New technologies for making vaccines. Vaccine 1999, 17:1596-1604.

9. Hutchings $\mathrm{CL}$, Gilbert SC, Hill AV, Moore AC: Novel protein and poxvirusbased vaccine combinations for simultaneous induction of humoral and cell-mediated immunity. J Immunol 2005, 175:599-606.

10. Janeway C: . Immunobiology: the immune system in health and disease New York: Garland Science 2005.

11. Loureiroa J, Ploegha HL: Antigen Presentation and the UbiquitinProteasome System in Host-Pathogen Interactions. Advances in Immunology 2006, 92:225-305.

12. Rock KL, Gramm C, Rothstein L, Clark K, Stein R, Dick L, Hwang D, Goldberg AL: Inhibitors of the proteasome block the degradation of 
most cell proteins and the generation of peptides presented on MHC class I molecules. Cell 1994, 78:761-771.

13. Kelly A, Powis SH, Kerr LA, Mockridge I, Elliott T, Bastin J, UchanskaZiegler B, Ziegler A, Trowsdale J, Townsend A: Assembly and function of the two $A B C$ transporter proteins encoded in the human major histocompatibility complex. 1992.

14. Lautscham G, Rickinson A, Blake N: TAP-independent antigen presentation on MHC class I molecules: Lessons from Epstein-Barr virus. Microbes and Infection 2003, 5:291-299.

15. Hansen $\mathrm{TH}$, Bouvier $\mathrm{M}: \mathrm{MHC}$ class I antigen presentation: learning from viral evasion strategies. Nat Rev Immunol 2009, 9:503-513.

16. Seliger B, Ritz U, Abele R, Bock M, Tampé R, Sutter G, Drexler I, Huber C, Ferrone $S$ : Immune escape of melanoma: first evidence of structural alterations in two distinct components of the MHC class I antigen processing pathway. Cancer Res 2001, 61:8647-8650.

17. Larsen MV, Nielsen M, Weinzierl A, Lund O: TAP-Independent MHC Class I Presentation. Current Immunology Reviews 2006, 2:233-245.

18. Stoltze L, Schirle M, Schwarz G, Schroter C, Thompson MW, Hersh LB, Kalbacher $\mathrm{H}$, Stevanovic S, Rammensee HG, Schild H: Two new proteases in the MHC class I processing pathway. Nat. Immunol 2000, 1:413-418.

19. Zhang GL, Petrovsky N, Kwoh CK, August JT, Brusic V: PRED(TAP): a system for prediction of peptide binding to the human transporter associated with antigen processing. Immunome Res 2006, 2:3.

20. Chen M, Bouvier M: Analysis of interactions in a tapasin/class I complex provides a mechanism for peptide selection. EMBO J 2007, 26:1681-1690.

21. Schoenhals GJ, Krishna RM, Grandea AG: Retention of empty MHC class I molecules by tapasin is essential to reconstitute antigen presentation in invertebrate cells. EMBO J 1999, 18:743, others.

22. Yewdell JW, Bennink JR: Immunodominance in major histocompatibility complex class I-restricted T lymphocyte responses. Annu Rev Immunol 1999, 17:51-88.

23. Moutaftsi M, Peters B, Pasquetto V, Tscharke DC, Sidney J, Bui HH, Grey H, Sette $A$ : A consensus epitope prediction approach identifies the breadth of murine T(CD8+)-cell responses to vaccinia virus. Nat Biotechnol 2006, 24:817-819.

24. Burrows SR, Rossjohn J, McCluskey J: Have we cut ourselves too short in mapping CTL epitopes? Trends Immunol 2006, 27:11-16.

25. Rammensee H, Bachmann J, Emmerich NP, Bachor OA, Stevanovic S: SYFPEITHI: database for MHC ligands and peptide motifs. Immunogenetics 1999, 50:213-219.

26. Falk K, Rotzschke O, Rammensee HG: Cellular peptide composition governed by major histocompatibility complex class I molecules. Nature 1990, 348:248-251

27. Kondo A, Sidney J, Southwood S, del Guercio MF, Appella E, Sakamoto H, Grey HM, Celis E, Chesnut RW, Kubo RT, Sette A: Two distinct HLA-A*0101specific submotifs illustrate alternative peptide binding modes. Immunogenetics 1997, 45:249-258

28. Kubo RT, Sette A, Grey HM, Appella E, Sakaguchi K, Zhu NZ, Arnott D, Sherman N, Shabanowitz J, Michel H: Definition of specific peptide motifs for four major HLA-A alleles. J Immunol 1994, 152:3913-3924.

29. Pamer $E G$, Davis $C E$, So M: Expression and deletion analysis of the Trypanosoma brucei rhodesiense cysteine protease in Escherichia coli. Infect Immun 1991, 59:1074-1078.

30. Rotzschke O, Falk K, Stevanovic S, Jung G, Walden P, Rammensee HG: Exact prediction of a natural T cell epitope. Eur J Immunol 1991, 21:2891-2894.

31. Sette A, Buus S, Appella E, Smith JA, Chesnut R, Miles C, Colon SM, Grey HM: Prediction of major histocompatibility complex binding regions of protein antigens by sequence pattern analysis. Proc Natl Acad Sci U S A 1989, 86:3296-3300.

32. Meister GE, Roberts CG, Berzofsky JA, De Groot AS: Two novel T cell epitope prediction algorithms based on MHC-binding motifs; comparison of predicted and published epitopes from Mycobacterium tuberculosis and HIV protein sequences. Vaccine 1995, 13:581-591.

33. Parker KC, Bednarek MA, Coligan JE: Scheme for ranking potential HLA-A2 binding peptides based on independent binding of individual peptide side-chains. J Immunol 1994, 152:163-175.

34. Stryhn A, Pedersen LO, Romme T, Holm CB, Holm A, Buus S: Peptide binding specificity of major histocompatibility complex class I resolved into an array of apparently independent subspecificities: quantitation by peptide libraries and improved prediction of binding. Eur I Immunol 1996, 26:1911-1918.
35. Schafer JR, Jesdale BM, George JA, Kouttab NM, De Groot AS: Prediction of well-conserved HIV-1 ligands using a matrix-based algorithm, EpiMatrix. Vaccine 1998, 16:1880-1884

36. Rammensee HG, Bachmann J, Stevanovic S: . MHC ligands and Peptide Motifs New York: Chapman \& Hall 1997.

37. Reche PA, Glutting JP, Reinherz EL: Prediction of MHC class I binding peptides using profile motifs. Hum Immunol 2002, 63:701-709.

38. Nielsen M, Lundegaard C, Worning P, Hvid CS, Lamberth K, Buus S, Brunak S, Lund O: Improved prediction of MHC class I and class II epitopes using a novel Gibbs sampling approach. Bioinformatics 2004, 20:1388-1397.

39. Peters B, Sette A: Generating quantitative models describing the sequence specificity of biological processes with the stabilized matrix method. BMC Bioinformatics 2005, 6:132

40. Bui HH, Sidney J, Peters B, Sathiamurthy M, Sinichi A, Purton KA, Mothe BR, Chisari FV, Watkins DI, Sette A: Automated generation and evaluation of specific MHC binding predictive tools: ARB matrix applications. Immunogenetics 2005, 57:304-314.

41. Lin HH, Ray S, Tongchusak S, Reinherz EL, Brusic V: Evaluation of MHC class I peptide binding prediction servers: applications for vaccine research. BMC Immunol 2008, 9:8.

42. Peters B, Bui HH, Frankild S, Nielson M, Lundegaard C, Kostem E, Basch D, Lamberth K, Harndahl M, Fleri W, Wilson SS, Sidney J, Lund O, Buus S, Sette A: A community resource benchmarking predictions of peptide binding to MHC-I molecules. PLoS Comput Biol 2006, 2:e65.

43. Adams HP, Koziol JA: Prediction of binding to MHC class I molecules. J Immunol Methods 1995, 185:181-190.

44. Brusic V, Rudy G, Harrison LC: Prediction of MHC binding peptides using artificial neural networks. Complex systems: mechanism of adaptation Amsterdam: IOS PressStonier RJ, Yu XS 1994, 253-260.

45. Buus $S$, Lauemoller SL, Worning $P$, Kesmir $C$, Frimurer T, Corbet $S$, Fomsgaard A, Hilden J, Holm A, Brunak S: Sensitive quantitative predictions of peptide-MHC binding by a 'Query by Committee' artificial neural network approach. Tissue Antigens 2003, 62:378-384.

46. Gulukota K, Sidney J, Sette A, DeLisi C: Two complementary methods for predicting peptides binding major histocompatibility complex molecules. J Mol Biol 1997, 267:1258-1267.

47. Nielsen M, Lundegaard C, Worning P, Lauemøller SL, Lamberth K, Buus S, Brunak S, Lund O: Reliable prediction of T-cell epitopes using neural networks with novel sequence representations. Protein Sci 2003, 12:1007-1017.

48. Dönnes P, Kohlbacher O: SVMHC: a server for prediction of MHC-binding peptides. Nucleic Acids Res 2006, 34:W194-W197.

49. Vita R, Zarebski L, Greenbaum JA, Emami H, Hoof I, Salimi N, Damle R, Sette A, Peters B: The immune epitope database 2.0. Nucleic Acids Res 2010, 38:D854-D862.

50. Sette A, Fleri W, Peters B, Sathiamurthy M, Bui HH, Wilson S: A roadmap for the immunomics of category A-C pathogens. Immunity 2005, 22:155-161.

51. Lundegaard C, Lund O, Buus S, Nielsen M: Major histocompatibility complex class I binding predictions as a tool in epitope discovery. Immunology 2010, 130:309-318.

52. Lundegaard $\mathrm{C}$, Lamberth $\mathrm{K}$, Harndahl M, Buus S, Lund O, Nielsen M: NetMHC-3.0: accurate web accessible predictions of human, mouse and monkey MHC class I affinities for peptides of length 8-11. Nucleic Acids Res 2008, 36:W509-W512.

53. Lundegaard C, Lund O, Kesmir C, Brunak S, Nielsen M: Modeling the adaptive immune system: predictions and simulations. Bioinformatics 2007, 23:3265-3275.

54. Toussaint NC, Kohlbacher O: Towards in silico design of epitope-based vaccines. Expert Opin. Drug Discov 2009, 4697

55. Lundegaard C, Lund O, Nielsen M: Accurate approximation method for prediction of class I MHC affinities for peptides of length 8,10 and 11 using prediction tools trained on 9mers. Bioinformatics 2008, 24:1397-1398.

56. Wang P, Sidney J, Dow C, Mothé B, Sette A, Peters B: A systematic assessment of MHC class II peptide binding predictions and evaluation of a consensus approach. PLoS Comput Biol 2008, 4:e1000048.

57. Sidney J, del Guercio MF, Southwood S, Engelhard VH, Appella E, Rammensee HG, Falk K, Rötzschke O, Takiguchi M, Kubo RT: Several HLA alleles share overlapping peptide specificities. J Immunol 1995, 154:247-259 
58. Lund $O$, Nielsen M, Kesmir C, Petersen AG, Lundegaard C, Worning P, Sylvester-Hvid C, Lamberth K, Røder G, Justesen S, Buus S, Brunak S: Definition of supertypes for HLA molecules using clustering of specificity matrices. Immunogenetics 2004, 55:797-810.

59. Doytchinova IA, Guan P, Flower DR: Identifiying human MHC supertypes using bioinformatic methods. J Immunol 2004, 172:4314-4323.

60. Reche PA, Reinherz EL: Definition of MHC supertypes through clustering of MHC peptide binding repertoires. Artificial Immune Systems, Proceedings Berlin: Springer Verlag 2004, 189-196.

61. Reche PA, Reinherz EL: Definition of MHC supertypes through clustering of MHC peptide-binding repertoires. Methods Mol Biol 2007, 409:163-173.

62. Sidney J, Grey HM, Southwood S, Celis E, Wentworth PA, del Guercio MF, Kubo RT, Chesnut RW, Sette A: Definition of an HLA-A3-like supermotif demonstrates the overlapping peptide-binding repertoires of common HLA molecules. Hum Immunol 1996, 45:79-93.

63. Sidney J, Peters B, Frahm N, Brander C, Sette A: HLA class I supertypes: a revised and updated classification. BMC Immunol 2008, 9:1.

64. Buus S, Stryhn A, Winther K, Kirkby N, Pedersen LO: Receptor-ligand interactions measured by an improved spun column chromatography technique. A high efficiency and high throughput size separation method. Biochim Biophys Acta 1995, 1243:453-460.

65. Harndahl M, Justesen S, Lamberth K, Røder G, Nielsen M, Buus S: Peptide binding to HLA class I molecules: homogenous, high-throughput screening, and affinity assays. J Biomol Screen 2009, 14:173-180.

66. Sidney J, Southwood S, Oseroff C, del Guercio MF, Sette A, Grey HM: Measurement of $\mathrm{MHC}$ /peptide interactions by gel filtration. Curr Protoc Immunol 2001, Chapter 18:Unit 18.3.

67. Sylvester-Hvid C, Kristensen N, Blicher T, Ferre H, Lauemoller SL, Wolf XA, Lamberth K, Nissen MH, Pedersen LO, Buus S: Establishment of a quantitative ELISA capable of determining peptide-MHC class I interaction. Tissue Antigens 2002, 59:251-258.

68. Zhang Q, Wang P, Kim Y, Haste-Andersen P, Beaver J, Bourne PE, Bui HH, Buus S, Frankild S, Greenbaum J, Lund O, Lundegaard C, Nielsen M, Ponomarenko J, Sette A, Zhu Z, Peters B: Immune epitope database analysis resource (IEDB-AR). Nucleic Acids Res 2008, 36:W513-W518.

69. Zhang GL, Khan AM, Srinivasan KN, August JT, Brusic V: MULTIPRED: computational system for prediction of promiscuous HLA binding peptides. Nucleic Acids Res 2005, 33:W172-W179.

70. Jacob L, Vert JP: Efficient peptide-MHC-I binding prediction for alleles with few known binders. Bioinformatics 2008, 24:358-366.

71. Jojic N, Reyes-Gomez M, Heckerman D, Kadie C, Schueler-Furman O: Learning MHC I-peptide binding. Bioinformatics 2006, 22:e227-e235.

72. Nielsen $M$, Lundegaard $C$, Blicher $T$, Lamberth $K$, Harndahl M, Justesen $S$, Røder G, Peters B, Sette A, Lund O, Buus S: NetMHCpan, a method for quantitative predictions of peptide binding to any $\mathrm{HLA}-\mathrm{A}$ and $-\mathrm{B}$ locus protein of known sequence. PLOS ONE 2007, 2:e796.

73. Hoof I, Peters B, Sidney J, Pedersen LE, Sette A, Lund O, Buus S, Nielsen M: NetMHCpan, a method for MHC class I binding prediction beyond humans. Immunogenetics 2009, 61:1-13.

74. Zhang H, Lundegaard C, Nielsen M: Pan-specific MHC class I predictors: a benchmark of HLA class I pan-specific prediction methods. Bioinformatics 2009, 25:83-89.

75. Kloetzel PM: The proteasome and MHC class I antigen processing Biochim Biophys Acta 2004, 1695:225-233.

76. Kloetzel PM: Generation of major histocompatibility complex class I antigens: functional interplay between proteasomes and TPPII. Nat Immunol 2004, 5:661-669.

77. Nielsen M, Lundegaard C, Lund O, Keșmir C: The role of the proteasome in generating cytotoxic T-cell epitopes: insights obtained from improved predictions of proteasomal cleavage. Immunogenetics 2005, 57:33-41.

78. Holzhutter $\mathrm{HG}$, Frommel C, Kloetzel PM: A theoretical approach towards the identification of cleavage-determining amino acid motifs of the $20 \mathrm{~S}$ proteasome. J. Mol. Biol. 1999, 286:1251-1265.

79. Holzhutter $\mathrm{HG}$, Kloetzel PM: A kinetic model of vertebrate $20 \mathrm{~S}$ proteasome accounting for the generation of major proteolytic fragments from oligomeric peptide substrates. Biophys $J 2000$ 79:1196-1205

80. Kuttler C, Nussbaum AK, Dick TP, Rammensee HG, Schild H, Hadeler KP: An Algorithm for the Prediction of Proteasomal Cleavages. J. Mol. Biol. 2000, 298:417-429.
81. Nussbaum AK, Kuttler C, Hadeler KP, Rammensee HG, Schild H: PAProC: a prediction algorithm for proteasomal cleavages available on the WWW. Immunogenetics 2001, 53:87-94.

82. Tenzer S, Stoltze L, Schonfisch B, Dengjel J, Muller M, Stevanovic S, Rammensee $H G$, Schild $H$ : Quantitative analysis of prion-protein degradation by constitutive and immuno-20S proteasomes indicates differences correlated with disease susceptibility. J Immunol 2004, 172:1083-1091.

83. Kesmir C, Nussbaum AK, Schild H, Detours V, Brunak S: Prediction of proteasome cleavage motifs by neural networks. Protein Eng 2002, 15:287-296.

84. Saxová P, Buus S, Brunak S, Kesmir C: Predicting proteasomal cleavage sites: a comparison of available methods. Int. Immunol 2003, 15:781-787.

85. Bhasin M, Raghava GPS: Pcleavage: an SVM based method for prediction of constitutive proteasome and immunoproteasome cleavage sites in antigenic sequences. Nucleic Acids Research 2005, 33:W202-W207.

86. Liu T, Liu W, Song Z, Jiao C, Zhu M, Wang X: Computational prediction of the specificities of proteasome interaction with antigen protein. Cell Mol Immunol 2009, 6:135-142.

87. Hanley JA, McNeil BJ: The meaning and use of the area under a receiver operating characteristic (ROC) curve. Radiology 1982, 143:29-36.

88. Ginodi I, Vider-Shalit T, Tsaban L, Louzoun Y: Precise score for the prediction of peptides cleaved by the proteasome. Bioinformatics 2008, 24:477-483.

89. Daniel S, Brusic V, Caillat-Zucman S, Petrovsky N, Harrison L, Riganelli D, Sinigaglia F, Gallazzi F, Hammer J, van Endert PM: Relationship between peptide selectivities of human transporters associated with antigen processing and HLA class I molecules. J Immunol 1998, 161:617-624.

90. Fruci D, Niedermann G, Butler RH, van Endert PM: Efficient MHC class Iindependent amino-terminal trimming of epitope precursor peptides in the endoplasmic reticulum. Immunity 2001, 15:467-476.

91. Peters B, Bulik S, Tampe R, Van Endert PM, Holzhütter HG: Identifying MHC class I epitopes by predicting the TAP transport efficiency of epitope precursors. J Immunol 2003, 171:1741-1749.

92. Lafuente EM, Reche PA: Prediction of MHC-peptide binding: a systematic and comprehensive overview. Curr Pharm Des 2009, 15:3209-3220.

93. Diez-Rivero CM, Chenlo B, Zuluaga P, Reche PA: Quantitative modeling of peptide binding to TAP using support vector machine. Proteins 2010, 78:63-72.

94. Tan TG, Mui E, Cong H, Witola WH, Montpetit A, Muench SP, Sidney J, Alexander J, Sette A, Grigg ME, Maewal A, McLeod R: Identification of T. gondii epitopes, adjuvants, and host genetic factors that influence protection of mice and humans. Vaccine 2010, 28:3977-3989.

95. Lankat-Buttgereit $B$, Tampe R: The transporter associated with antigen processing TAP: structure and function. FEBS Lett 1999, 464:108-112.

96. Sieker F, Straatsma TP, Springer S, Zacharias M: Differential tapasin dependence of MHC class I molecules correlates with conformational changes upon peptide dissociation: a molecular dynamics simulation study. Mol Immunol 2008, 45:3714-3722.

97. Lundegaard C, Nielsen M, Lund O: The validity of predicted T-cell epitopes. Trends Biotechnol 2006, 24:537-538.

98. Stranzl T, Larsen MV, Lundegaard C, Nielsen M: NetCTLpan: pan-specific MHC class I pathway epitope predictions. Immunogenetics 2010, 62:357-368.

99. Hakenberg J, Nussbaum AK, Schild H, Rammensee HG, Kuttler C, Holzhutter HG, Kloetzel PM, Kaufmann SH, Mollenkopf HJ: MAPPP: MHC class I antigenic peptide processing prediction. Appl Bioinformatics 2003 2:155-158.

100. Larsen MV, Lundegaard C, Lamberth K, Buus S, Brunak S, Lund O, Nielsen M: An integrative approach to CTL epitope prediction: a combined algorithm integrating MHC class I binding, TAP transport efficiency, and proteasomal cleavage predictions. Eur J Immunol 2005, 35:2295-2303.

101. Tenzer S, Peters B, Bulik S, Schoor O, Lemmel C, Schatz MM, Kloetzel PM, Rammensee HG, Schild H, Holzhutter HG: Modeling the MHC class I pathway by combining predictions of proteasomal cleavage, TAP transport and MHC class I binding. Cell Mol Life Sci 2005, 62:1025-1037.

102. Doytchinova IA, Guan P, Flower DR: EpiJen: a server for multistep T cell epitope prediction. BMC Bioinformatics 2006, 7:131.

103. Donnes $P$, Kohlbacher $O$ : Integrated modeling of the major events in the MHC class I antigen processing pathway. Protein Sci 2005, 14:2132-2140. 
104. Larsen MV, Lundegaard C, Lamberth K, Buus S, Lund O, Nielsen M: Largescale validation of methods for cytotoxic T-lymphocyte epitope prediction. BMC Bioinformatics 2007, 8:424.

105. Perez CL, Larsen MV, Gustafsson R, Norstrom MM, Atlas A, Nixon DF Nielsen M, Lund O, Karlsson AC: Broadly immunogenic HLA class I supertype-restricted elite CTL epitopes recognized in a diverse population infected with different HIV-1 subtypes. J Immunol 2008, 180:5092-5100.

106. Wang M, Lamberth K, Harndahl M, Røder G, Stryhn A, Larsen MV, Nielsen M, Lundegaard C, Tang ST, Dziegiel MH, Rosenkvist J, Pedersen AE, Buus S, Claesson MH, Lund O: CTL epitopes for influenza A including the H5N1 bird flu; genome-, pathogen-, and HLA-wide screening. Vaccine 2007, 25:2823-2831.

107. Cohen S: Cell mediated immunity and the inflammatory system. Human pathology 1976, 7:249.

108. Castellino F, Zhong G, Germain RN: Antigen presentation by MHC class II molecules: invariant chain function, protein trafficking, and the molecular basis of diverse determinant capture. Hum Immunol 1997, 54:159-169.

109. Nielsen M, Lund O, Buus S, Lundegaard C: MHC Class II epitope predictive algorithms. Immunology 2010, 130:319-328.

110. Stern LJ, Brown JH, Jardetzky TS, Gorga JC, Urban RG, Strominger JL, Wiley DC: Crystal structure of the human class II MHC protein HLA-DR1 complexed with an influenza virus peptide. Nature 1994, 368:215-221.

111. Rudensky AY, Preston-Hurlburt P, Hong SC, Barlow A, Janeway CA: Sequence analysis of peptides bound to MHC class II molecules. Nature 1991, 353:622-627

112. Hammer J, Bono E, Gallazzi F, Belunis C, Nagy Z, Sinigaglia F: Precise prediction of major histocompatibility complex class II-peptide interaction based on peptide side chain scanning. J Exp Med 1994, 180:2353-2358.

113. Singh $H$, Raghava GP: ProPred: prediction of HLA-DR binding sites. Bioinformatics 2001, 17:1236-1237.

114. Bhasin M, Raghava GP: SVM based method for predicting HLADRB1*0401 binding peptides in an antigen sequence. Bioinformatics 2004, 20:421-423.

115. Godkin AJ, Smith KJ, Willis A, Tejada-Simon MV, Zhang J, Elliott T, Hill AV: Naturally processed HLA class II peptides reveal highly conserved immunogenic flanking region sequence preferences that reflect antigen processing rather than peptide-MHC interactions. J Immunol 2001, 166:6720-6727.

116. Chang ST, Ghosh D, Kirschner DE, Linderman JJ: Peptide length-based prediction of peptide-MHC class II binding. Bioinformatics 2006, 22:2761-2767.

117. Nielsen M, Lundegaard C, Lund O: Prediction of MHC class II binding affinity using SMM-align, a novel stabilization matrix alignment method. BMC Bioinformatics 2007, 8:238.

118. Lin HH, Zhang GL, Tongchusak S, Reinherz EL, Brusic V: Evaluation of MHCIl peptide binding prediction servers: applications for vaccine research. BMC Bioinformatics 2008, 9(Suppl 12):S22.

119. Nielsen M, Lund $\mathrm{O}$ : NN-align. An artificial neural network-based alignment algorithm for MHC class II peptide binding prediction. BMC Bioinformatics 2009, 10:296.

120. Nielsen M, Lundegaard C, Blicher T, Peters B, Sette A, Justesen S, Buus S, Lund O: Quantitative predictions of peptide binding to any HLA-DR molecule of known sequence: NetMHCIlpan. PLoS Comput Biol 2008, 4 : e1000107.

121. Rosa DS, Ribeiro SP, Cunha-Neto E: CD4+ T cell epitope discovery and rational vaccine design. Arch Immunol Ther Exp (Warsz) 2010, 58:121-130.

122. Mustafa AS: Th1 cell reactivity and HLA-DR binding prediction for promiscuous recognition of MPT63 (Rv1926c), a major secreted protein of Mycobacterium tuberculosis. Scand J Immunol 2009, 69:213-222.

123. Fischer W, Perkins S, Theiler J, Bhattacharya T, Yusim K, Funkhouser R, Kuiken C, Haynes B, Letvin NL, Walker BD, Hahn BH, Korber BT: Polyvalent vaccines for optimal coverage of potential T-cell epitopes in global HIV1 variants. Nat Med 2007, 13:100-106.

124. Toussaint NC, Dönnes P, Kohlbacher O: A mathematical framework for the selection of an optimal set of peptides for epitope-based vaccines. PLOS Comput Biol 2008, 4:e1000246.
125. Toussaint NC, Kohlbacher O: OptiTope-a web server for the selection of an optimal set of peptides for epitope-based vaccines. Nucleic Acids Res 2009, 37:W617-W622

126. Hildner K, Edelson BT, Purtha WE, Diamond M, Matsushita H, Kohyama M, Calderon B, Schraml BU, Unanue ER, Diamond MS: Batf3 deficiency reveals a critical role for CD8 alpha\}+ dendritic cells in cytotoxic T cell immunity. Science 2008, 322:1097, others.

127. Lamberth K, Røder G, Harndahl M, Nielsen M, Lundegaard C, SchaferNielsen C, Lund O, Buus S: The peptide-binding specificity of HLA-A*3001 demonstrates membership of the HLA-A3 supertype. Immunogenetics 2008, 60:633-643.

128. Hoof I, Pérez CL, Buggert M, Gustafsson RK, Nielsen M, Lund O, Karlsson AC: Interdisciplinary Analysis of HIV-Specific CD8+ T Cell Responses against Variant Epitopes Reveals Restricted TCR Promiscuity. J Immunol 2010.

129. Rapin N, Hoof I, Lund O, Nielsen M: MHC motif viewer. Immunogenetics 2008, 60:759-765.

doi:10.1186/1745-7580-6-S2-S3

Cite this article as: Lundegaard et al:: State of the art and challenges in sequence based T-cell epitope prediction. Immunome Research 20106 (Suppl 2):S3.

\section{Submit your next manuscript to BioMed Central and take full advantage of:}

- Convenient online submission

- Thorough peer review

- No space constraints or color figure charges

- Immediate publication on acceptance

- Inclusion in PubMed, CAS, Scopus and Google Scholar

- Research which is freely available for redistribution 